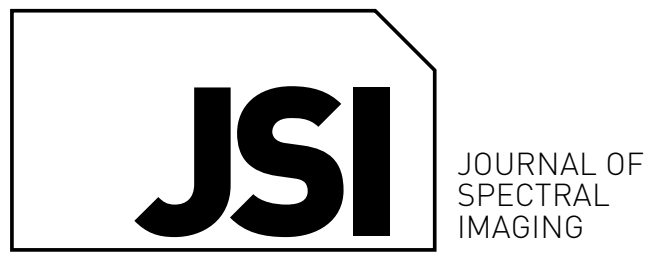

Paper Presented at HSI 2014, Hyperspectral Imaging and Applications, Coventry, UK

\title{
openaccess
}

\section{Advances in hyperspectral remote sensing I: The visible Fourier transform hyperspectral imager}

\author{
J. Bruce Rafert \\ Professor of Physics, Department of Physics, North Dakota State University, Fargo, USA
}

We discuss early hyperspectral research and development activities during the 1990s that led to the deployment of aircraft and satellite payloads whose heritage was based on the use of visible, spatially modulated, imaging Fourier transform spectrometers, beginning with early experiments at the Florida Institute of Technology, through successful launch and deployment of the Visible Fourier Transform Hyperspectral Imager on MightySat II.1 on 19 July 2000. In addition to a brief chronological overview, we also discuss several of the most interesting optical engineering challenges that were addressed over this timeframe, present some as-yet un-exploited features of field-widened (slit-less) SMIFTS instruments, and present some images from ground-based, aircraft-based and satellitebased instruments that helped provide the impetus for the proliferation and development of entire new families of instruments and countless new applications for hyperspectral imaging.

Keywords: hyperspectral, visible Fourier transform imager, precision agriculture, transportation infrastructure

\section{Introduction}

The past $~ 50$ years has seen what can now in hindsight best be described as the debut of a new metadiscipline-hyperspectral remote sensing. Taxonomy previously confined for use by a small and restricted number of specialists has now penetrated virtually every scientific and engineering discipline from agriculture through zoology, although routine use of what has become a very mature technology is not yet ubiquitous across all fields.

Figure 1 depicts the growth of the field, as measured by one readily available metric-how many papers have the word hyperspectral as indexed by Google Scholar. The timeframe can be divided into three main intervals: pre-1990 when use of "hyperspectral" was constant and extremely sparse; the decade of the 1990s when a period of exponential growth occurred; and the post-2000 period with a high linear trajectory to over 10,000 occurrences per year. This paper focusses on just one of many specific technologies (Visible, Fourier

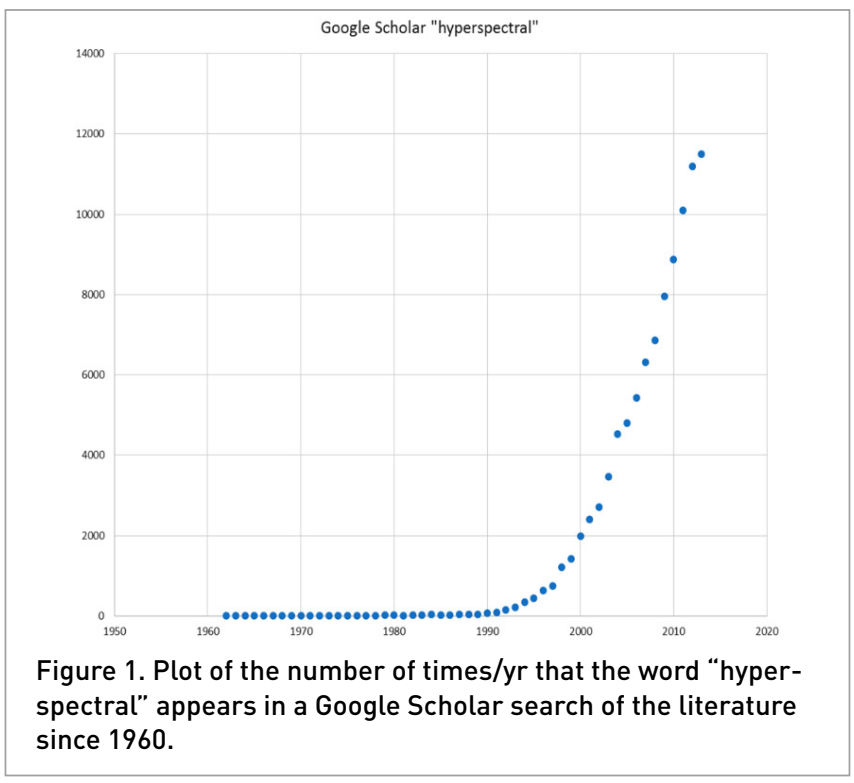


transform Hyperspectral Imaging-VFTHSI) that contributed to the early growth of the field during the decade of the 1990s, when numerous investigators and laboratories were experimenting and developing a wide array of hyperspectral instrument configurations (e.g., dispersive, Fourier Transform, acousto-optical) in a number of band passes (visible, SWIR, LWIR) for an array of applications. In this paper we will trace the development of just the VFTHSI instrument design heritagea subset of Spatially Modulated Imaging Fourier Transform Spectrometers (SMIFTS) which also saw the development and impacts of some notable LWIR instruments (Lucey, 1992), from initial brass board implementation to orbit. Topics will include a short review of operational features as seen from the perspective of an optical systems engineer, tracking of the design heritage from brass board to orbit, some observational data from several of the instrument variations and a short summary of unexplored vistas for the future.

\section{VFTHSI basic operational characteristics}

Figure 2 depicts the now well-known configuration of a VFTHSI instrument. Shown is a single, polychromatic ray from a target spatial element $(x, y)$. The ray passes through a fore optic (not shown) with focal length and f/ratio such that infinity focus is at the field stop, which serves only as a spatial mask in the $\mathrm{x}$-direction (but allows image formation in the y-direction). We note that the spatial mask serves primarily to aid in image reconstruction following data reduction, although the wider the spatial mask, the more photons that are admitted to the system-the limiting case being elimination of the field stop entirely. The ray passes through some type of a beam splitter, and hence through the triangle-path or Sagnac interferometer. We also make note that deviations from $R=T=0.5$ split of the ray by the beam splitter across the entire band pass result in rapid deterioration of fringe visibility on the detector, particularly when the instrument is configured for operation near the Nyquist sampling limit. The Sagnac has a number of desirable features including extreme ease of configuration and align-

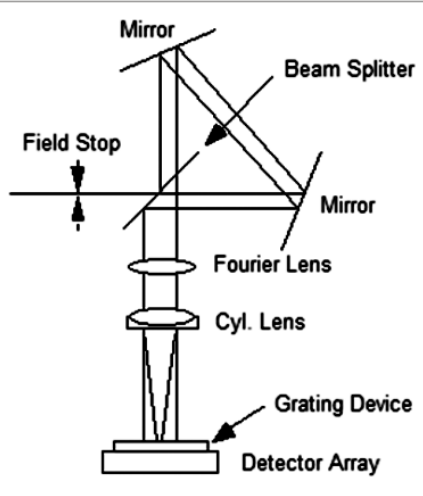

Moiré \& Grating Configuration

Figure 2. Triangle path interferometer. ment, and is amenable to monolithic solutions to avoid vibration or misalignment issues. The lower-right mirror in Figure 2 is shown with an offset $s$ from the triangle orientation which introduces a sheer in the two mutually coherent parallel rays shown exiting the interferometer downward.

Those two rays enter a Fourier optic, whose focal length is selected to be the distance back through the interferometer to the field stop. Arbitrary magnification le.g., size of the aperture function necessary to fully illuminate a CCD of size $n \times m$ ) can be achieved via selection of $f /$ ratio with the fore optic. A cylinder lens whose focal length is chosen to reimage the target $y$-dimension onto the visible $C C D$ has no power in the (target) $x$-dimension. The net effect of this optical device for a ray of wavenumber $k$ is that an interferogram, $/(x)=$ $\int\left(I_{0}(k) / 2\right) \times[1+\cos (2 \pi k s)] d k$, is created on the CCD detector in the (target) $x$-direction, while the target $y$-direction creates a one-dimensional image orthogonal to the interferogram on the CCD. A transmission grating (not discussed in this paper) can be placed above the detector, or at other locations in the interferometer, to achieve shifts of the recovered spectrum from the interferogram that more fully cover spatial frequency space.

\section{Tracking the design heritage from brass board to orbit}

Our original experiments with the VFTHSI were undertaken on a surplus $2 \times 3$ foot brass board at the Florida Institute of Technology-using available surplus beam splitters and surplus optical table components-essentially, nothing more sophisticated than the optics shown in Figure 2. An early version of the brass board was mounted aboard the R1 and R2 telescopes at the Malabar Test Facility, where proof of concept hypercubes were obtained for space objects (space station MIR) as well as cooperative ground-based targets on the Malabar optical range (readily available eight-bit video cameras and image intensifiers were used as the CCD sensors for these early experiments). Concurrently, a utility study was undertaken as part of a larger project funded through Darpa with collaborators from the University of Hawaii and the University of Central Florida. ${ }^{1}$ Interestingly, the final report which alluded to "dozens or hundreds" of potential applications for hyperspectral imaging was received with some scepticism, perhaps feeling that such proliferation of applications of such an seemingly limited technology would never occur.

Early success with such modest hardware and demonstration of utility quickly led the US Air Force to provide strong development support for more sophisticated instruments. Figure 3 shows the 3D optical schematic of the Kestrel/ Michigan Technological University (MTU) VFTHSI., ${ }^{2,3}$

The design heritage of the original prototype is retained, although the need to reduce chromatic correction across the band pass led to a number of refinements in the design. The beam splitter is no longer square, becoming more compact 


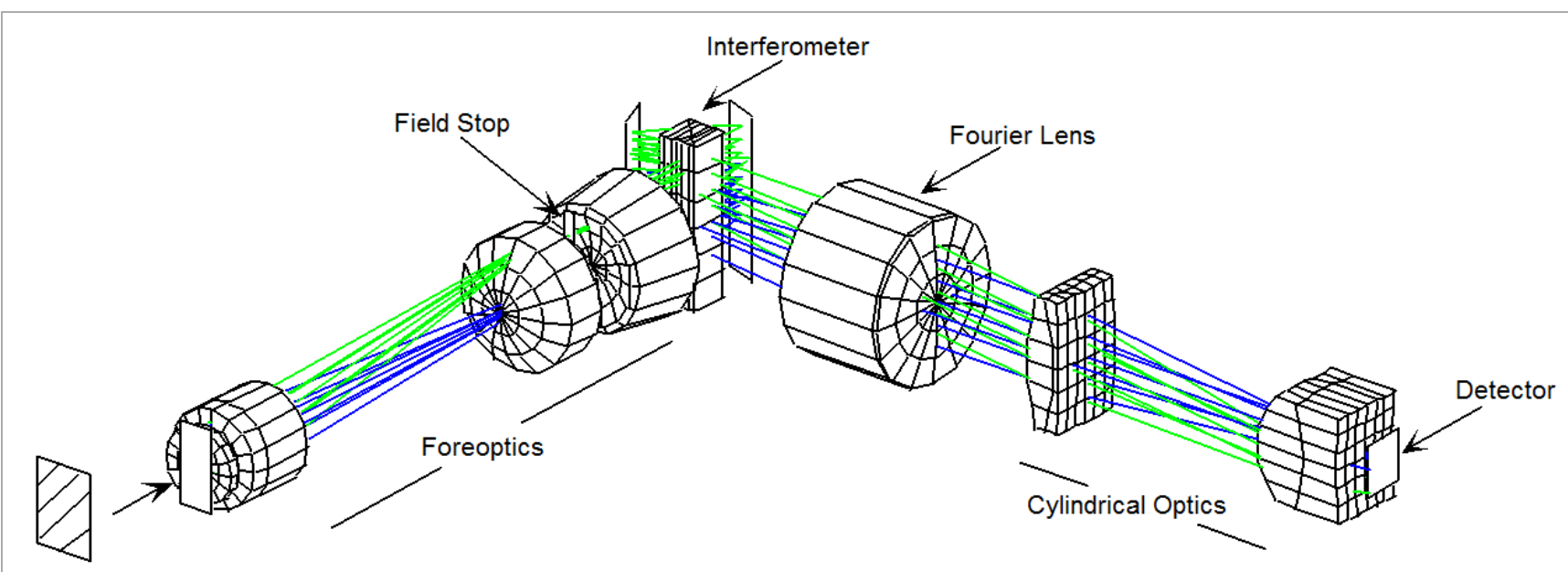

Scene

Figure 3. The Kestrel/MTU VFTHSI Raytrace.

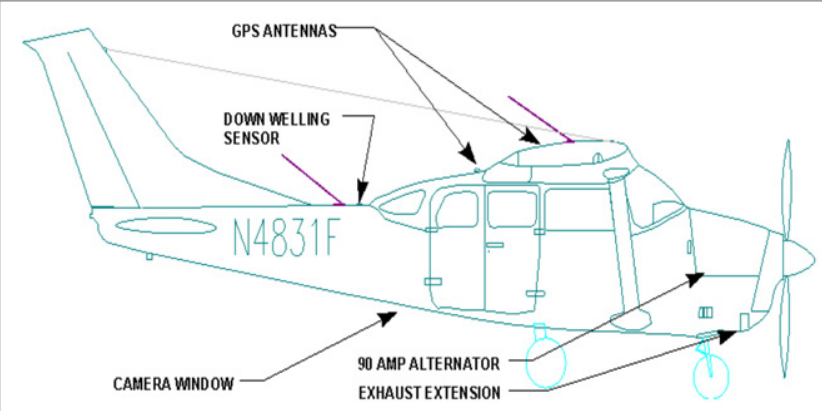

Figure 4. Kestrel Cessna.

and rectangular (with the larger dimension in the target $y$-direction). Curiously, we found that the Fourier optic could be positioned partially in front of and partially behind the beam splitter-a significant saving in space. A variety of designs which incorporated reflective and/or torroidal optics were also studied.

This updated version of the VFTHSI was built in duplicateone system was integrated into the Kestrel Cessna shown in Figure 4, while the second (optically identical) was fabricated as a scanning ground based unit at MTU.

Figure 5(a) is one flight line of the Kestrel VFTHSI over the MTU campus, while Figure 5(b) depicts spectral profiles for seven different targets within the scene (the spectral profiles shown here are uncorrected for sensor or atmospheric effects).

Figure 6 shows a schematic of the version of VFTHSI that was built to fly in space on MightySat II.1.4.5 This was the first hyperspectral satellite that returned a hyperspectral image from space. Note the introduction of square reflective fore optics (weight saving), and monolithic components (to address

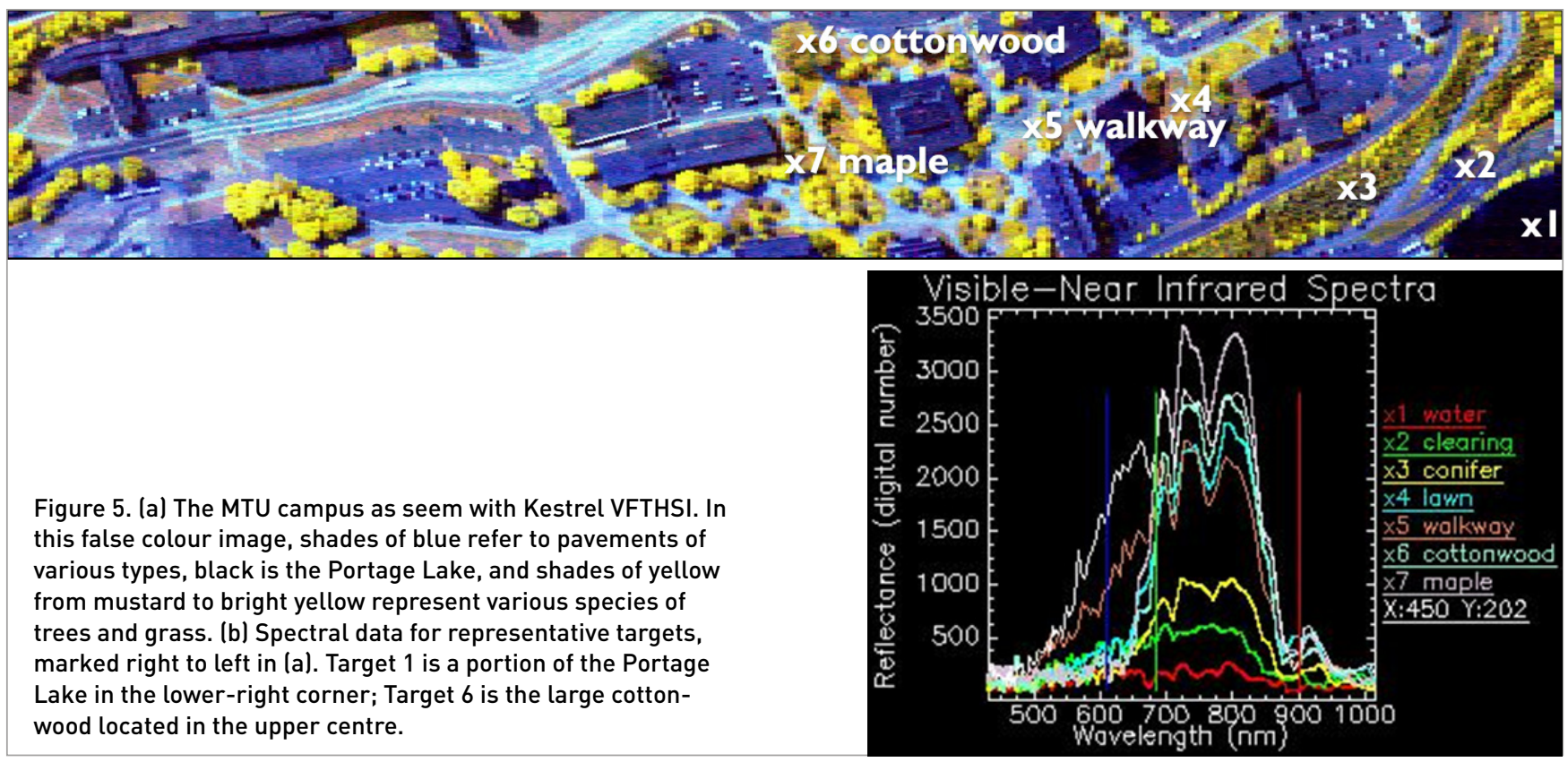




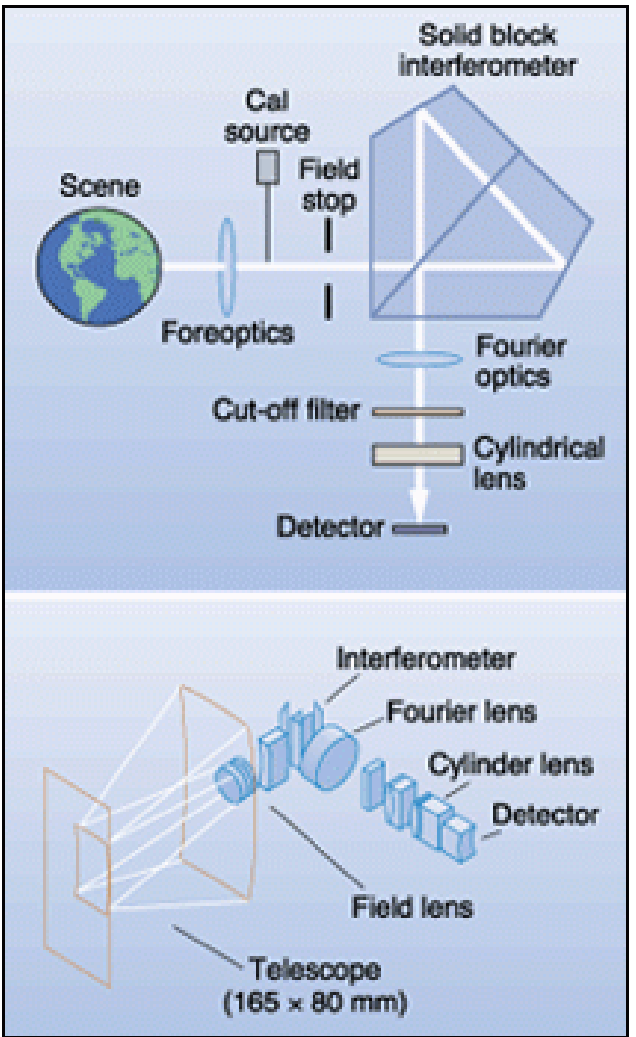

Figure 6. Schematic of the version of VFTHSI that flew in space on MightySat II.1. @ 2001 PennWell Corporation. Reproduced from Reference 5 with permission.

vibration and alignment issues during launch and on-orbit operations).

\section{Summary and emerging vistas}

As seen from the perspective of 2015 , there is now little doubt that hyperspectral remote sensing is in the process of becoming a major remote sensing tool which is being utilised by researchers and practitioners in virtually every scientific and engineering discipline. Recent papers show a broad array of applications where hyperspectral is being applied to predicting beef eating quality, ${ }^{6}$ adulteration levels of lamb, detection of foreign materials in powdered foods, ${ }^{8}$ mineral exploration, ${ }^{9}$ estimation of lead concentrations in flooded soils, ${ }^{10}$ detection of trace elements of allergens, ${ }^{11}$ spatial spectral imaging of water content in mushrooms and hydration of food products, ${ }^{12}$ small scale hydrothermal alteration (work by Harris ${ }^{13}$ and many other areas well beyond traditional remote sensing, surveillance and target detection). Capable, light weight, low-power hyperspectral sensor systems are now readily available from multiple vendors. ${ }^{14}$ Recent developments of high throughput, video rate, ${ }^{15,16}$ plenoptic and/or field-widened tomographic imagers ${ }^{17,15}$ offer a number of new sensor options. One example is a high throughput, slit-less FT instrument, in which two-dimensional spatial intensity variations are modulated by the intensity variation of a white light interferogram on a row by row basis. ${ }^{18}$ Such an approach has the major advantage of increased throughput (the slit does not block any of the aperture function), while requiring only deconvolution of the two intensity distributions via a push broom vector.

Two particular areas-precision agriculture and transportation infrastructure including road, rail and pipelines-are in an extremely rapid initial phase of experimentation and adoption. We also make note of a significant recent interest from other wide ranging areas including medicine ${ }^{19}$ and artwork inspection. ${ }^{20}$ Our current efforts are focused on optimising hyperspectral remote sensing for use with lightweight (less than 50 pounds) unmanned aircraft systems (UAS) and to provide the relevant training necessary for future practitioners to construct and deploy full solutions that include the sensor system, data storage and archiving, and an appropriate data decision framework. Several hundred relevant hotlinks pertaining to hyperspectral imaging of the transportation infrastructure are available and provided elsewhere. ${ }^{21,22}$

\section{Acknowledgements}

We gratefully and enthusiastically acknowledge numerous conversations, collaborations and the many significant contributions that Joel Blatt, Paul Lucey, Tom Rusk, Glenn Sellar, Eirik Holbert, Harold Newby, Susan Durham, John Otten, Andrew Meigs, Gene Butler, Chris Gittins, Mark North, Raj Bridgelall, Jinchang Ren and Stephen Marshall have made to the efforts described here, and also to their many other contributions to the growth of the field of hyperspectral imaging.

\section{References}

1. P.G. Lucey, T. Williams, K. Horton, K. Hinck, J.B. Rafert and E.T. Rusk, "SMIFTS: A cryogenically cooled infrared spatially modulated imaging Fourier transform spectrometer for remote sensing applications", Proceedings of the ISSSR 1, 251 (1992).

2. L.J. Otten, E.W. Butler, J.B. Rafert and R.G. Sellar, "The design of an airborne Fourier transform visible hyperspectral imaging system for light aircraft environmental remote sensing", Aerosense '95, Orlando, Florida (1995).

3. J.B. Rafert, W. Slough, A. Pilant, L.J. Otten, A. Meigs, A. Jones and E. Butler, "MTU-Kestrel airborne hyperspectral imaging campaigns of the Lake Superior ecosystem", Proc. SPIE 3753, Imaging Spectrometry V, 486 (1999). doi: http://dx.doi.org/ 10.1117/12.366271

4. L.J. Otten, J.B. Rafert, A. Meigs, B. Jones, R. Prinzing, R. Fronterhouse, R.G. Sellar and P. Hodge, "Engineering model for the MightySat II.1 hyperspectral imager", Proc. SPIE 3221, Sensors, Systems, and Next- 
Generation Satellites, 412 (1997). doi: http://dx.doi.org/ $\underline{10.1117 / 12.298108}$

5. J.B. Rafert, L.J. Otten, A.D. Meigs and E.W. Butler, "Orbiting Fourier transform hyperspectral sensor", Laser Focus 21 February (2001). http://www.laserfocusworld.com/articles/print/volume-37/issue-5/features/ hyperspectral-imaging/satellite-sends-hyperspectralimages-from-space.html

6. T. Qiao, J. Ren, C. Craigie, J. Zabalza, C. Maltin and S. Marshall, "Comparison between near infrared spectroscopy and hyperspectral imaging in predicting beef eating quality", HSI 2014 Hyperspectral Imaging and Applications, Coventry, UK (2014).

7. Y. Pu, Y. Feng, M. Kamruzzaman and D. Sun, "Quantification of adulteration levels in minced lamb meat using NIR hyperspectral imaging", HSI 2014 Hyperspectral Imaging and Applications, Coventry, UK (2014).

8. A. Herrero-Langreo, P. Mishra, J. Roger, N. Gorretta, L. Lleo, B. Diezma and P. Barreiro, "Multispectral imaging for detection of foreign food material in powder food", HSI 2014 Hyperspectral Imaging and Applications, Coventry, UK (2014).

9. M. Gagnon, P. Tremblay, S. Savary, M. Duval, V. Farley, P. Laguex and M. Chamberland, "Airborne thermal infrared hyperspectral imaging for mineral exploration”, HSI 2014 Hyperspectral Imaging and Applications, Coventry, UK (2014).

10. S. Lamine, G. Petropoulos, P. Brewer, C. Kalaqitzidis, K. Manevski, M. Macklin and W. Haresign, "Investigating the potential of hyperspectral imaging (HSI) for the quantitative estimation of lead contamination in soil (LCS)", HSI 2014 Hyperspectral Imaging and Applications, Coventry, UK (2014).

11. P. Mishra, A. Herrero-Langreo, P. Barreiro, J.M. Roger, B. Diezma, N. Gorretta and L. Lleó, “Detection and quantification of peanut traces in wheat flour by NIR hyperspectral imaging spectroscopy using principal- component analysis", J. Near Infrared Spectrosc. 23, 15 (2015). doi: http://dx.doi.org/ 10.1255/jnirs.1141

12. A. Gowen, "The role of water in NIR hyperspectral imaging of biological materials", HSI 2014 Hyperspectral Imaging and Applications, Coventry, UK (2014).
13. J. Harris, I. Crawford and C. Cousins, "Endmember subspace estimation algorithms applied to hyperspectral images of small scale hydrothermal alteration", HSI 2014 Hyperspectral Imaging and Applications, Coventry, UK (2014).

14. K. Lynch and S. Hill, "Miniaturized hyperspectral sensor for UAV applications”, HSI 2014 Hyperspectral Imaging and Applications, Coventry, UK (2014).

15. A. Harvey, J. Ramos, L. Brewer and A. Gorman, “Videorate multispectral imaging: Application to microscopy and macroscopy", HSI 2014 Hyperspectral Imaging and Applications, Coventry, UK (2014).

16. J. Jafolla, M. Dambrowski, S. Henry and S. Briggs, "Hyperspectral to multispectral: Video rate spectral imaging applications", HSI 2014 Hyperspectral Imaging and Applications, Coventry, UK (2014).

17. 0 . Pust and H. Fabricus, "Linear variable filters for biomedical and hyperspectral imaging applications", HSI 2014 Hyperspectral Imaging and Applications, Coventry, UK (2014).

18. A. Barducci, F. Castagnoli, D. Guzzi, P. Marcoionni and I. Pippi, The ALISEO instrument: Calibration methods and assessment of interferometer response", in Geolnformation in Europe, Ed by M.A. Gomarasca. Millpress, Netherlands (2007). ISBN 9789059660618

19. C. Blanch-Perez-del-Notario, N. Nguyen-Do-Trong, W. Saeys and A. Lambrechts, "Hyperspectral analysis for extraction of chemical characteristics in dehydrated bones", HSI 2014 Hyperspectral Imaging and Applications, Coventry, UK (2014).

20. V. Papadakis, R. Groves, E. Ribes-Gomez, V. Tornari, C. Portales, A. Borreman, J. Vermeiren and J. Ward, "A novel 3D-hyperspectral imaging device for artwork inspection and deterioration estimation-the SYDDARTA project", HSI 2014 Hyperspectral Imaging and Applications, Coventry, UK (2014).

21. R. Bridgelall and J. Rafert, http://www.ugpti.org/smartse/research/citations/.

22. J.B. Rafert, "Before hyperspectral ruled the world", HSI 2014 Hyperspectral Imaging and Applications, Coventry, UK (2014). 